\title{
Photoacoustic imaging using an 8-beam Fabry-Perot scanner
}

\author{
Nam Huynh, Olumide Ogunlade, Edward Zhang, Ben Cox, Paul Beard \\ Department of Medical Physics and Biomedical Engineering, University College London, Gower \\ Street, London WC1E 6BT, UK
}

\begin{abstract}
The planar Fabry Perot (FP) photoacoustic scanner has been shown to provide high resolution 3D images of soft tissue structures in vivo. However a significant limitation of current embodiments of the concept is low image acquisition speed. To address this, a novel multi-beam scanner architecture has been developed. This enables 8 interrogation beams to be scanned simultaneously across the FP sensor and the photoacoustic signals detected in parallel. In addition, an excitation laser operating at $200 \mathrm{~Hz}$ was used. The combination of parallelising the detection and the high pulse repetition frequency (PRF) of the excitation laser has enabled significant reductions in image acquisition time to be achieved. A 3D image can now be acquired in 10 seconds and 2D images at near video rates are possible.
\end{abstract}

Keywords: Photoacoustic tomography, Fabry Perot sensor

\section{INTRODUCTION}

The well-established Fabry-Perot ultrasound sensor can provide high resolution 3D photoacoustic images of tissue structures [1]. The sensor is a transparent Fabry-Perot (FP) etalon comprising a polymer film spacer sandwiched between a pair of mirrors. Acoustically-induced changes in the optical thickness of the spacer modulate the reflectivity of the etalon which can be detected by measuring the changes in the reflected power of an incident laser beam [2-4]. By interrogating the sensor with a scanning focussed laser beam, it is possible to synthesise arrays of many tens of thousands of points with small element sizes. However, the combination of the sequential nature of the sensor read-out and the low PRF of most OPO based excitation laser systems results in long acquisition times. For example, a typical 2D scan comprising 10,000 A-lines over a 10mm x 10mm scan area takes approximately 4 minutes using an excitation laser with a PRF of $50 \mathrm{~Hz}$.

This paper presents a novel multi-beam scanner architecture which enables 8 interrogation beams to be scanned simultaneously across the FP sensor and the photoacoustic signals detected in parallel. In addition, a custom OPO laser system that provides a higher PRF $(200 \mathrm{~Hz})$ than most commercially available equivalents was used as the excitation source. The combination of the parallelisation of the sensor read-out and the high PRF of the excitation laser provides a significant reduction in acquisition time. To demonstrate the fast imaging capability of the system, images of a dynamic phantom and the subcutaneous blood vessels in the human palm have been obtained.

Photons Plus Ultrasound: Imaging and Sensing 2016, edited by Alexander A. Oraevsky, Lihong V. Wang Proc. of SPIE Vol. 9708, 97082L · @ 2016 SPIE · CCC code: 1605-7422/16/\$18 · doi: 10.1117/12.2214334 


\section{EXPERIMENTAL SETUP}

Figure 1 shows a schematic of the 8 beam scanner. The output of a fibre coupled $1550 \mathrm{~nm}$ interrogation source is split equally between 8 fibres which are connected to an 8-beam bundle. A photodiode transimpedance amplifier configuration with DC- and AC-coupled outputs is connected to the return port of each circulator in order to detect the reflected beams. The DC-coupled outputs are connected to a 200kS/s 16-bit analog-to-digital (A/D) card within the PC and used to record the interferometer transfer function (ITF). The AC-coupled outputs are connected to an 8-channel $60 \mathrm{MHz}$ digitizing card and used to record the time varying reflected optical power modulation produced by the incident acoustic wave. An X-Y optical scanner comprising a pair of mutually orthogonal closed loop galvanometer mirrors was used to scan the 8-beams across the FP sensor head.

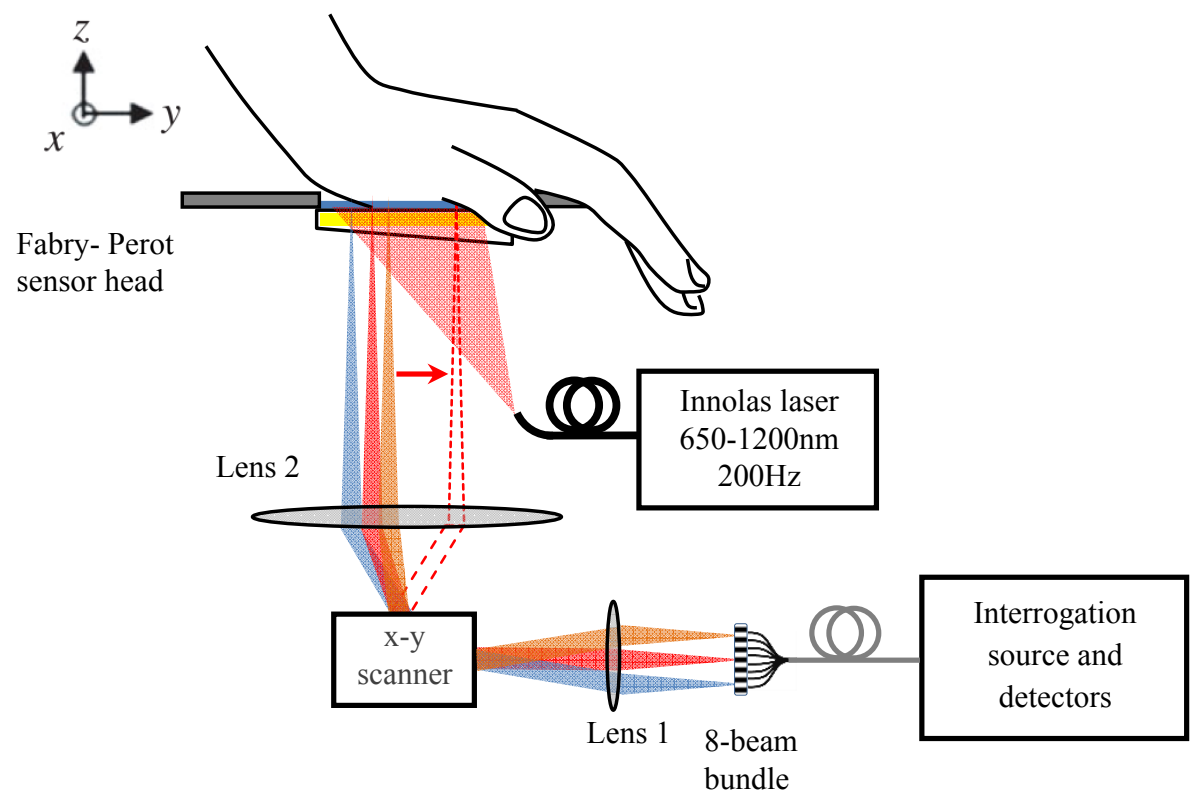

Figure 1 Schematic of the multi-beam scanner

The beam spacing at the sensor surface is approximately $330 \mu \mathrm{m}$. In order to achieve a higher scanning resolution, an interleaved scanning scheme was applied, e.g. 2 interleaved positions were used in all the experiments on this study to achieve a spatial sampling interval of $110 \mu \mathrm{m}$. The excitation laser was a custom-designed, table-top 532nm pumped OPO $(660 \mathrm{~nm}-1300 \mathrm{~nm})$ operating at a PRF of $200 \mathrm{~Hz}$ and providing pulse energies in the range $10-15 \mathrm{~mJ}$ depending on wavelength. 


\section{RESULTS}

\subsection{D images}

\section{a. Dynamic phantom}

To create a dynamic phantom, a series of highly absorbing dots were painted on to an optical fibre which was inserted into a polythene tube $(0.58 \mathrm{~mm}$ inner diameter, $0.96 \mathrm{~mm}$ outer diameter $)$ containing heavily diluted $(\sim 2 \%)$ Indian ink. The tube containing the optical fibre was submersed in a 1\% Intralipid suspension and arranged as shown in figure 2a. Motion was induced by manually pulling the fibre out of the tube in the direction indicated by the red arrow. A series of 2D photoacoustic images of the moving fibre were acquired by repeatedly scanning the interrogation laser beams back and forth along a line parallel to the optical fibre. The length of the line-scan was $10 \mathrm{~mm}$ and the spatial sampling interval was $110 \mu \mathrm{m}$. The images were reconstructed offline after acquiring the whole dataset. Figure $2 \mathrm{~b}$ shows selected $2 \mathrm{D}$ photoacoustic images. Three successive images (0:04.07s to 0:04.20s) illustrate a group of moving dots as the fibre was pulled. The last image $(0: 16.13 \mathrm{~s})$ shows the ink filled polythene tube more clearly when the fibre was completely withdrawn. The image frame rate in this experiment was approximately $17 \mathrm{~Hz}$.

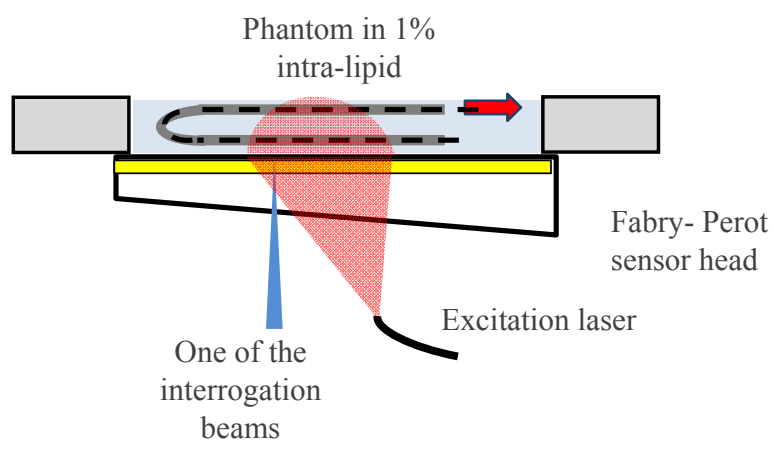

(a)

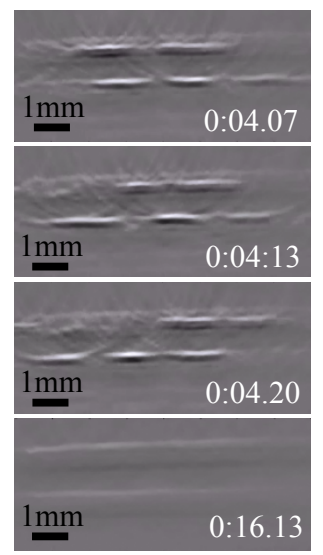

(b)

Figure 2 2D imaging of a dynamic phantom comprising a series of highly absorbing dots painted on to an optical fibre inserted within a dye filled tube immersed in Intralipid. (a) Experimental setup and (b) photoacoustic images. Movie1: dot_painted_fibre is available online. Image frame rate $=17 \mathrm{~Hz}$.

\section{b. Human wrist}

In this experiment, 2D images of the blood vessels in the human wrist were obtained whilst the wrist was rotated with respect to the scan-line. The length of the line scan was $13.2 \mathrm{~mm}$ and the step size was $110 \mu \mathrm{m}$ steps corresponding to 120 scanning points. The excitation wavelength was $720 \mathrm{~nm}$. Figure 3a shows the volunteer's wrist during the experiment and the line scan position. Figure $3 \mathrm{~b}$ shows selected 2D photoacoustic images illustrating the high contrast exhibited by several superficial blood vessels. The image frame rate was approximately $13 \mathrm{~Hz}$ 
(a)

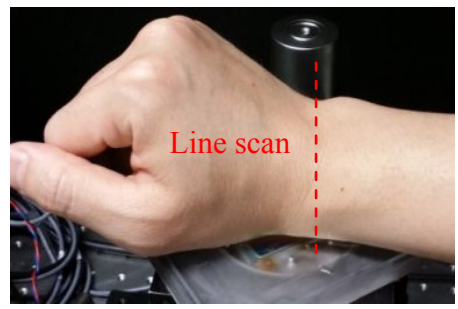

(b)
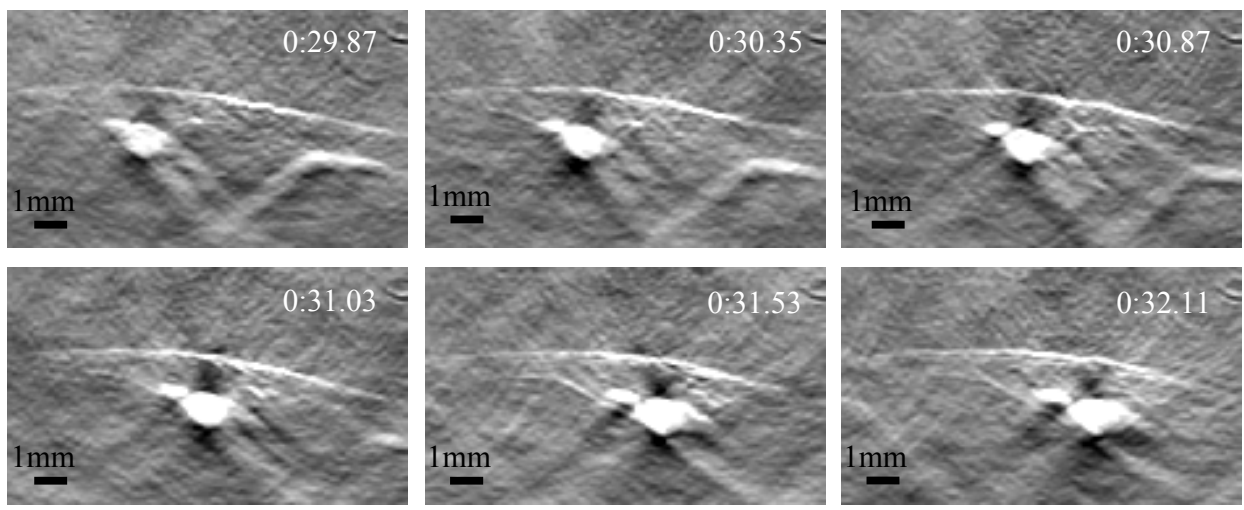

Figure 3 2D images of a human wrist (a) Orientation of line-scan; (b) series of photoacoustic images. Movie2: wristscan is available online. Image frame rate $=13 \mathrm{~Hz}$

\subsection{In vivo 3D images of human palm}

A 3D in vivo image of the subcutaneous vasculature in the palm of a volunteer was obtained. The photoacoustic signals were mapped by scanning the 8 beams over an area of $10 \mathrm{~mm} \times 10 \mathrm{~mm}$ in steps of $110 \mu \mathrm{m}$. The time taken to acquire this data (8264 A-lines) was approximately 10 seconds. Figure 4a shows the hand resting on the FP sensor during experiments. Figure $4 \mathrm{~b}$ shows MIPs of the 3D image data set. The excitation wavelength was $850 \mu \mathrm{m}$.
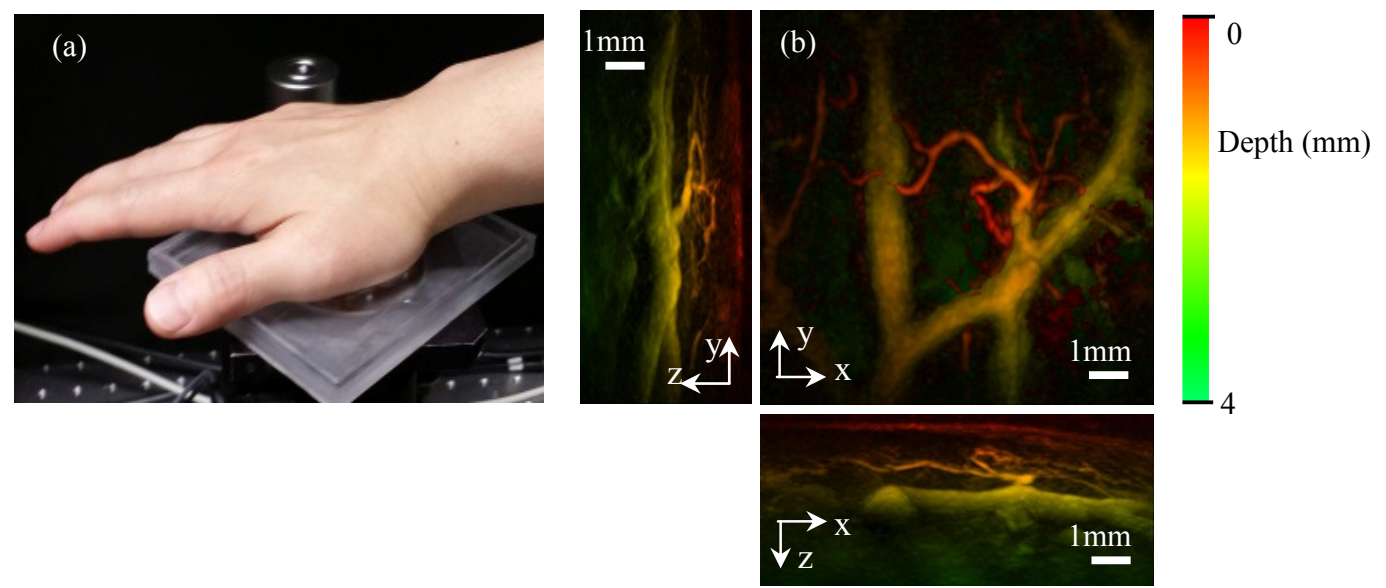

Figure 4 3D photoacoustic imaging of the human palm. (a) Photograph of the palm resting on the FP sensor head. (b) MIPs of the 3D image data set. 


\section{CONCLUSION}

A novel multi-beam scanner architecture that employs 8 equally spaced interrogation beams in order to parallelise the FP sensor read-out has been developed. Together with the high PRF of the excitation laser $(200 \mathrm{~Hz})$, this new system enabled significant reductions in image acquisition time to be achieved; 3D images of the human palm that would take in excess of 4 minutes using the first generation FP scanner (1) were obtained within 10 seconds and 2D images of the blood vessels in the human wrist were acquired at near video rates.

\section{ACKNOWLEDGEMENTS}

The authors acknowledge support from the Engineering and Physical Sciences Research Council (EP/K009745/1), UK and European Union project FAMOS (FP7 ICT, Contract 317744). The authors are grateful for the support of Christian Menhard of Innolas GmBH who developed the $200 \mathrm{~Hz}$ OPO laser system.

\section{REFERENCES}

[1] Jathoul AP, Laufer J, Ogunlade O, Treeby B, Cox B, Zhang E, Johnson P, Pizzey AR, Philip B, Marafioti T, Lythgoe MF, Pedley RB, Pule MA and Beard P, "Deep in vivo photoacoustic imaging of mammalian tissues using a tyrosinasebased genetic reporter", Nature Photonics 9, 239-246 (2015)

[2] Zhang E., Laufer J., \& Beard P., "Backward-mode multiwavelength photoacoustic scanner using a planar Fabry-Perot polymer film ultrasound sensor for high-resolution three-dimensional imaging of biological tissues", Appl. Opt. 47, 561577 (2008)

[3] Laufer J., Norris F., Cleary J., Zhang E., Treeby B., Cox B., Johnson P., Scambler P., Lythgoe M., Beard P., "In vivo photoacoustic imaging of mouse embryos", J Biomed Opt. 17(6), 061220 (2012)

[4] Beard P., "Biomedical photoacoustic imaging", Interface Focus, 1(4), pp.602-631(2011)

[5] Zhang EZ, Laufer JG, Pedley RB, Beard PC (2009) “In vivo high-resolution 3D photoacoustic imaging of superficial vascular anatomy", Physics in Medicine and Biology, 54, 1035-1046

[6] Zhang EZ, Povazay B, Laufer J, Alex A, Hofer B, Pedley B, Glittenberg C, Treeby B, Cox B, Beard P, and Drexler W, "Multimodal photoacoustic and optical coherence tomography scanner using an all optical detection scheme for 3D morphological skin imaging”, Biomedical Optics Express, 2(8), 2202-2215 (2011) 